\title{
Measurement of the Spatial Distribution of Neutrons in an Accelerator Room by the Combination of Activation Detectors and an Imaging Plate
}

\author{
Kazuyoshi Masumoto, Akihiro Toyoda, Kazuyoshi Eda and Toyoyuki Ishihara \\ Radiation Science Center, High Energy Accelerator Research Organization \\ Received June 6, 2002; accepted September 20, 2002
}

In order to measure the spatial distribution of neutrons in an accelerator room, the combined use of activation detectors and an imaging plate was tried. Activation detectors were set on monitoring points in the accelerator room. After the end of operation, the detectors were collected and pasted on the corresponding monitoring points on a layout plan of the accelerator room. The plan was overlaid with an imaging plate to develop the activity of activation detectors. This method is suitable for the simultaneous measurement of the activities of many detectors without any decay correction. The obtained image data are very useful for grasping the propagation of neutrons from the beamloss points and to make an effective design for reducing the activation of the accelerator components and accelerator buildings.

Key words: activation detector, imaging plate, neutron, accelerator, spatial distribution.

\section{Introduction}

The activation of accelerators, peripheral devices and shields has been a serious problem for the radiation protection of accelerator facilities in cases of maintenance and dismantling. ${ }^{1-3)}$ The primary beam hits various accelerator components during acceleration and transport. The radioactivity induced by the primary beam is high, but the activated area is limited. From their beam-loss points, neutrons are secondarily emitted in all directions by nuclear reactions, and some of them cause the activation of the surrounding materials such as the magnets, frames, cables, electrical circuits, tubes, vacuum pumps or the construction of buildings. Although the radioactivity caused by neutrons is low, it is not negligible, and the activated area is very wide. Therefore, measuring the neutron flux is necessary to estimate the degree of neutron activation of the accelerator and the structure of the building. The obtained results aid to design an effective shield and to propose a manual of beam transport to reduce the activation of the accelerator components and the surrounding materials.

Neutrons are continuously monitored with a BF-3 proportional counter or a He-3 proportional counter. This method

Correspondence to K. Masumoto

Radiation Science Center, High Energy Accelerator Research Organization,

Oho, Tsukuba, 305-0801, Japan is useful to detect the temporal variation of the dose rate. The cumulative dose of neutrons is also monitored with a film badge, a thermoluminescence detector and a fission track detector using CR-39 plastic. These methods, however, are not suitable to obtain the spatial distribution of neutrons. Neutrons can also be monitored by the activation detector method. In this method, the radioactivity induced in each detector has to be measured one by one. Therefore, the activity measurement and calculation are time-consuming and tedious, which results in a limitation of the number of detectors treated in one experiment. Recently, an imaging plate (IP) has been developed as a new radiography technique. ${ }^{4-7)}$ For example, the IP was applied to measure the distribution of the radioactivity near the surface of various natural products or the slightly contaminated surface. ${ }^{8)}$ One of the authors used activation detectors and the IP to monitor the bremsstrahlung and photoneutron profiles radiated from a Pt-target bombarded by an electron linear accelerator. ${ }^{9)}$ Activation detectors were polyethylene film, nickel, gold and aluminum foils for the detection of bremsstrahlung and fast neutrons by using the ${ }^{12} \mathrm{C}(\gamma, \mathrm{n}){ }^{11} \mathrm{C},{ }^{58} \mathrm{Ni}(\gamma, \mathrm{n}){ }^{57} \mathrm{Ni},{ }^{197} \mathrm{Au}(\gamma, \mathrm{n}){ }^{196} \mathrm{Au}$ and ${ }^{27} \mathrm{Al}(\mathrm{n}, \alpha){ }^{24} \mathrm{Na}$ reactions, respectively. The beam profile in-

TEL: 0298-64-5491 FAX: 0298-64-1993 e-mail: kazuyoshi.masumoto@kek.jp 
side the irradiation chamber was clearly obtained as a function of the distance from the Pt-target by analyzing the IP after being contacted with activation detectors.

It was not sure that the above method was applicable to detect the low flux of neutrons in the accelerator room. Then, we applied this method to measure the spatial distribution of neutrons in an accelerator room in this work. This technique is performed as follows: (1) each activation detector is set on a monitoring point in the accelerator room during operation, (2) each detector is collected and pasted on the monitoring point of a plan of the accelerator room after irradiation, (3) the plan is directly overlapped on the IP to develop the radioactivity of all monitoring points, (4) the latent image of all detectors is read out with an imaging analyzer simultaneously and (5) the overlapped picture of a image data and the plan are composed on a computer display.

\section{Experimental}

\section{Setting and measurement of the activation detector}

The neutron flux was measured at the SF-Cyclotron, which belongs to the Center for Nuclear Study, University of Tokyo, and is located in Tanashi Branch, High Energy Accelerator Research Organization, Tokyo.

Small tips of gold foil ( $11 \mathrm{~mm} \varnothing$ and $12 \mu \mathrm{m}$ in thickness) were used for monitoring the neutron flux as activation detectors, and the radioactivity of ${ }^{198} \mathrm{Au}$ induced by the ${ }^{197} \mathrm{Au}(\mathrm{n}, \gamma){ }^{198} \mathrm{Au}$ reaction was measured.

Activation detectors were set on the floor and walls of the accelerator room when the cyclotron was operated under different particles and energies, such as protons of $25 \mathrm{MeV}$ and deuterons of $30 \mathrm{MeV}$. The maximum number of monitoring points was 47 . The vertical distribution of the neutron flux along with the beam-transport line was also monitored by the activation detectors, which were hung on a tape strained from the ceiling to the floor at intervals of $50 \mathrm{~cm}$.

After the end of operation, the activation detectors were collected and pasted on the monitoring points of each detector on the plan. The IP (BAS III, Fuji Photo Film Co.) was overlaid with the plan to measure the activities of the detectors simultaneously. After an appropriate exposure time, the IP was scanned with a bio-imaging analyzer (BAS-1000, Fuji Photo Film Co.). A color image of the activity of the activation detectors was obtained as a two-dimensional map by the software (MacBAS, provided by FUJIX Co.). The intensity of the photo-stimulated luminescence in the unit of PSL was calculated automatically for the position of each activation detector. The image data was superimposed on the plan of the accelerator room on a computer display.

\section{Relationship between activity and PSL intensity}

In order to obtain the conversion factor from PSL intensity to the activity of ${ }^{198} \mathrm{Au}$ in $\mathrm{Bq}$, nine selected gold foils were measured. An automatic gamma-ray spectrometer, which comprises a Ge-detector (Model-7229P, Canberra), a multichannel analyzer (TRUMP, SEIKO EG\&G) and a small robot (Movemaster EX, Mitsubishi Electric Co.) for sample changing, was used for $\gamma$-ray measurements. The counting time was changed from 2000 to $10000 \mathrm{sec}$ for each activation detector. Each foil was set on a Ge-detector surface during measurements.

The PSL of gold foils was measured with the bio-imaging analyzer (BAS -1000) after gold foils were contacted with the IP for a certain exposure time. The exposure time was changed from 2 to $60 \mathrm{~min}$.

\section{Results and discussion}

\section{Selection of Activation detector and flux calculation}

As activation detectors, $\mathrm{In}, \mathrm{Au}, \mathrm{Co}, \mathrm{Mn}$ and $\mathrm{Na}$ have been used for thermal neutrons and $\mathrm{Al}, \mathrm{Bi}$ and $\mathrm{C}$ for fast neutrons. In this work, Au foils were chosen to monitor thermal neutrons because of its high sensitivity.

The flux was calculated based on the assumption that the resonance reaction is dominant, and its neutron capture crosssection is 1550 barn. It was confirmed that the activity of ${ }^{196} \mathrm{Au}$ induced by the ${ }^{197} \mathrm{Au}(\mathrm{n}, 2 \mathrm{n}){ }^{196} \mathrm{Au}$ reaction was negligibly small, and was barely observed at the position of the maximum neutron flux near the cyclotron.

\section{Uniformity in sensitivity on IP}

The homogeneous sensitivity of the IP should be confirmed in advance, because PSL values of various positions on the IP were compared with one another. After the IP was uniformly irradiated by an X-rays, the PSL intensity per unit area $\left(\mathrm{PSL} / \mathrm{mm}^{2}\right)$ were measured at six random sampling points. The results were in good agreement with one another, and their \% RSD was $4.1 \%$. Therefore, the detection sensitivity did not depend on the position of the IP.

\section{Relationship between the activity and PSL}

In order to obtain the conversion factor from PSL intensity to the activity of ${ }^{196} \mathrm{Au}$, nine gold foils, which contained radioactivity ranging from 15 to $200 \mathrm{~Bq}$ of ${ }^{198} \mathrm{Au}$, were set on the IP. The exposure times were also changed from 2 to $60 \mathrm{~min}$. The obtained results are shown in Fig. 1. The PSL linearly increased in proportion to the activity and the exposure time. The PSL of ${ }^{198} \mathrm{Au}$ activity could be measured within 3 to $5 \%$ RSD for exposure times of 10 to $20 \mathrm{~min}$. Then, the 


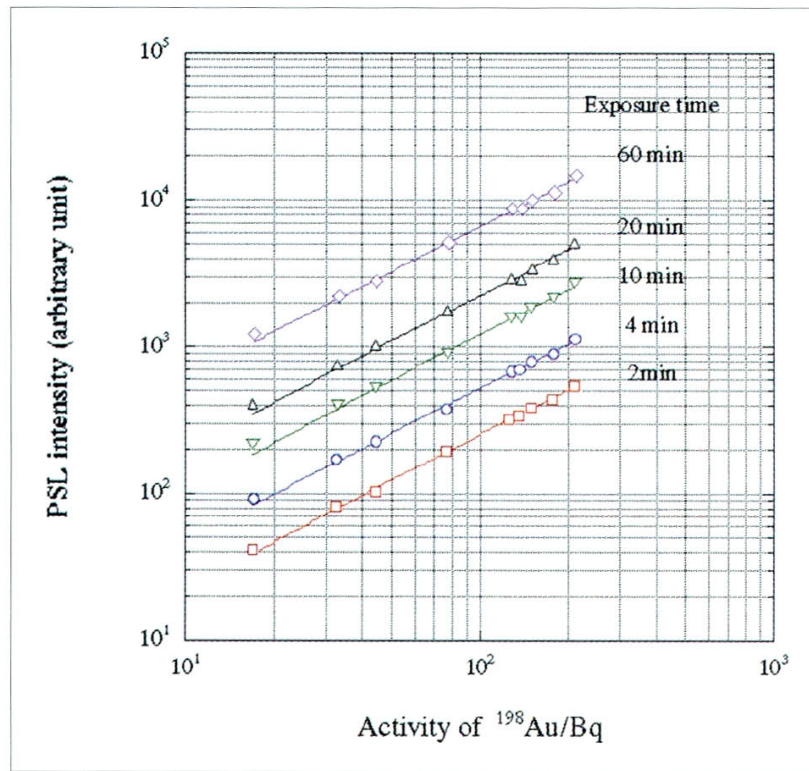

Fig. 1. Relationship between $\mathrm{Bq}$ of ${ }^{198} \mathrm{Au}$ and the PSL value under different exposure times.

exposure time was set on 20 min throughout this work. An empirical relation for ${ }^{198} \mathrm{Au}, \mathrm{PSL}=1.11 \times($ activity, $\mathrm{Bq}) \times$ (exposure times, min), was obtained.

\section{Spatial distribution of neutrons in the cyclotron room}

Fig. 2 shows the result of an experiment, where the proton energy was $25 \mathrm{MeV}$ and the average beam current was $2 \mu \mathrm{A}$. The beam was transported to the upper part of this figure. It was found that the neutrons were not uniformly distributed in the cyclotron room. The colored spots show the measuring positions of the activation detector. The color means the activity of ${ }^{198} \mathrm{Au}$; for instance, orange corresponds to the neutron flux of the order of $10^{3} / \mathrm{cm}^{2} / \mathrm{sec}$ and blue means $10^{2}$ $/ \mathrm{cm}^{2} / \mathrm{sec}$. The neutron flux near the deflector of the cyclotron was much higher than that of the opposite side of the beam extraction window. It was confirmed that neutrons were mainly produced near the deflector of the cyclotron. The neutron flux decreased with the distance from the cyclotron. It was confirmed that this method was useful to measure the neutron flux. In order to obtain more data simultaneously,

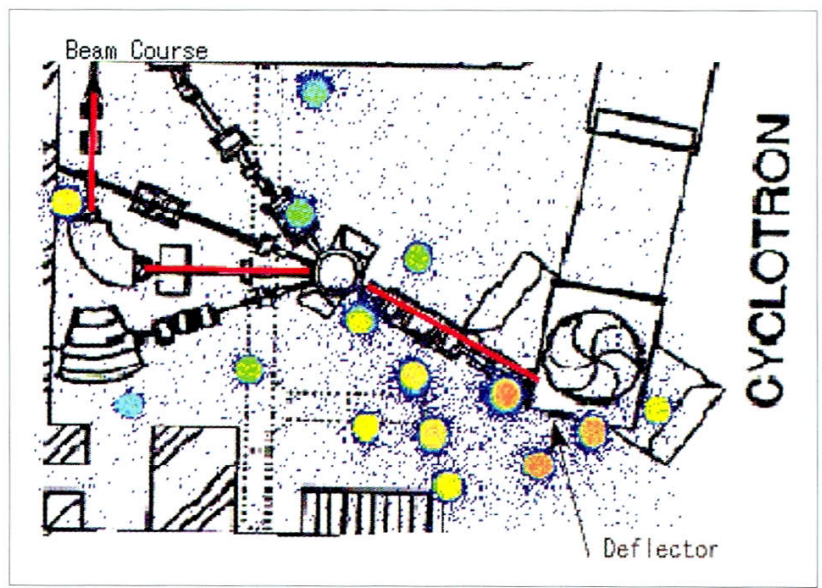

Fig. 2. Spatial distribution of the neutrons observed in the case of $25-\mathrm{MeV}$ proton acceleration.

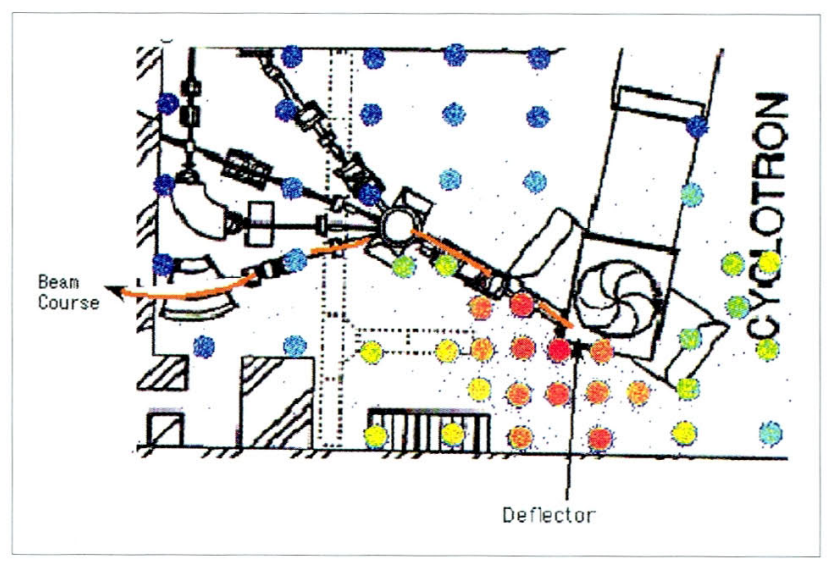

Fig. 3. Spatial distribution of the neutrons observed in the case of $30-\mathrm{MeV}$ deuteron acceleration.

about 50 samples were positioned on the floor and walls of the cyclotron room as shown in Fig. 3. The deuteron energy was $30 \mathrm{MeV}$ and the beam was transported to the left side of this figure. The neutron fluence in case of deuteron beam was higher than that in case of proton beam. Table 1 gives the minimum and maximum values of the neutron flux obtained in the case of deuteron acceleration. The difference between the maximum flux and the minimum flux was

Table 1. Minimum and Maximum flux of neutrons observedin the case of 30-MeV deuteron irradiation.

\begin{tabular}{ccccccc}
\hline & Weight/mg & Au atoms & PSL & Activity/Bq & Activity ${ }^{\star} / \mathrm{Bq}$ & Neutron flux ${ }^{\star \star}$ \\
\hline Min & 21.8 & $6.66 \mathrm{E}+19$ & 235.1 & $8.50 \mathrm{E}+00$ & $1.10 \mathrm{E}+01$ & $7.38 \mathrm{E}+02$ \\
Max & 22.3 & $6.81 \mathrm{E}+19$ & 3359 & $1.20 \mathrm{E}+02$ & $1.60 \mathrm{E}+02$ & $1.03 \mathrm{E}+04$ \\
\hline
\end{tabular}

*; Activity just after irradiation

$* *$; unit $=\mathrm{n} \bullet \mathrm{cm}^{-2} \bullet \mathrm{sec}^{-1}$ 


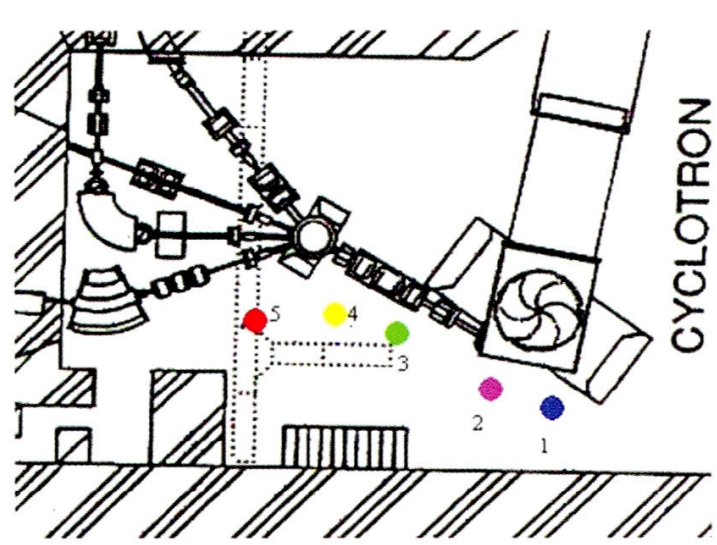

(a) Monitoring positions

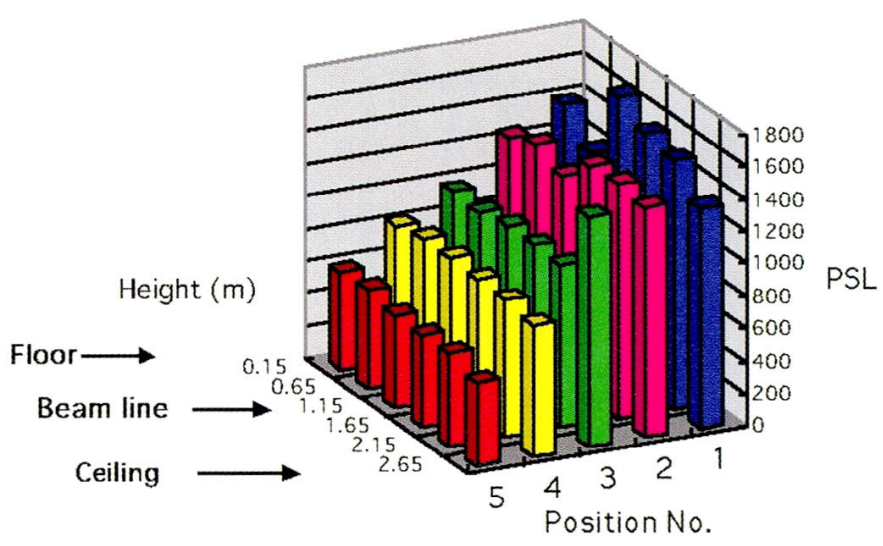

(b) PSL values

Fig. 4. Vertical change of neutrons obtained at five positions. (a) Measuring positions, (b) the PSL values observed.

about one order.

\section{Vertical distribution of neutrons in the cyclotron room}

The vertical distribution of neutrons was measured in the same manner. The five measurement positions were selected between the cyclotron and the switch magnet, as shown in Fig. 4 (a). Six activation detectors were hung on a string from the ceiling at intervals of $50 \mathrm{~cm}$. The heights of monitors were $0.15,0.65,1.15,1.65,2.15$ and $2.65 \mathrm{~m}$ from the floor and the beam line was $1.15 \mathrm{~m}$ from the floor. The observed result is shown in Fig. 4(b). The thermal neutron flux gradually decreased with the distance from the cyclotron. On the other hand, no vertical dependence of the thermal neutron fluence could be observed. This might be explained as meaning that the fast neutrons emitted from the beam loss point in the cyclotron were gradually thermalized in their flights and reflected by the floor and the ceiling. Therefore, the thermal part of the neutron fluence might become almost uniform in the vertical direction. This result should be confirmed by comparing with a theoretical calculation of the neutron propagation.

\section{Comparison with the induced radioactivity in concrete in the cyclotron room}

The radioactivity induced in the concrete wall was measured and compared with the result of a neutron-flux measurement. Concrete samples were obtained at various points on the floor, wall and ceiling, and measured with a Ge-detector for analyzing the residual radioactivity. The major radionuclides were ${ }^{60} \mathrm{Co}$ and ${ }^{152} \mathrm{Eu}$, the elemental abundances of which were about 10 and $1 \mu \mathrm{g} / \mathrm{g}$, respectively. Fig. 5 shows the specific activity of ${ }^{60} \mathrm{Co}$ in the concrete of the cyclotron building as an example. The distribution of ${ }^{60} \mathrm{Co}$ activity was quite similar to the that of neutron-flux observed in Fig. 2 and 3 . The maximum activity of ${ }^{60} \mathrm{Co}$, which was observed near the deflector of the cyclotron, was $0.059 \mathrm{~Bq} / \mathrm{g}$. In order to calculate the activity of ${ }^{60} \mathrm{Co}$, following conditions were assumed. The epithermal and thermal neutron flux during operation was $10^{4} \mathrm{n} / \mathrm{cm}^{2} / \mathrm{sec}$, respectively. The cyclotron has been operated for 20 years and 100 days per year. The thermal and epithermal cross-section of ${ }^{59} \mathrm{Co}$ are 37 and 74 barn. The concentration of $\mathrm{Co}$ is $10 \mu \mathrm{g} / \mathrm{g}$. The obtained value was $0.06 \mathrm{~Bq} / \mathrm{g}$, which quite agreed with the observed maximum value, $0.059 \mathrm{~Bq} / \mathrm{g}$.

Such an information is very useful to reduce an activation of the floor and wall by covering the higher flux region with a boron.

\section{Conclusion}

The activation detector method is very effective for measuring neutrons emitted by beam loss in an accelerator room. In order to grasp the spatial distribution of neutron fluence, the combined use of activation detectors and an IP was very useful, because the activity of many pieces of detector can be measured simultaneously. The PSL value is directly converted to the activity in $\mathrm{Bq}$ without any decay correction by using the empirical relation. A neutron flux of $10^{3} \mathrm{n} / \mathrm{cm}^{2} / \mathrm{sec}$ could be observed at an exposure time of $10 \mathrm{~min}$ within a few \% of RSD.

One of the most important goals of radiation safety con- 

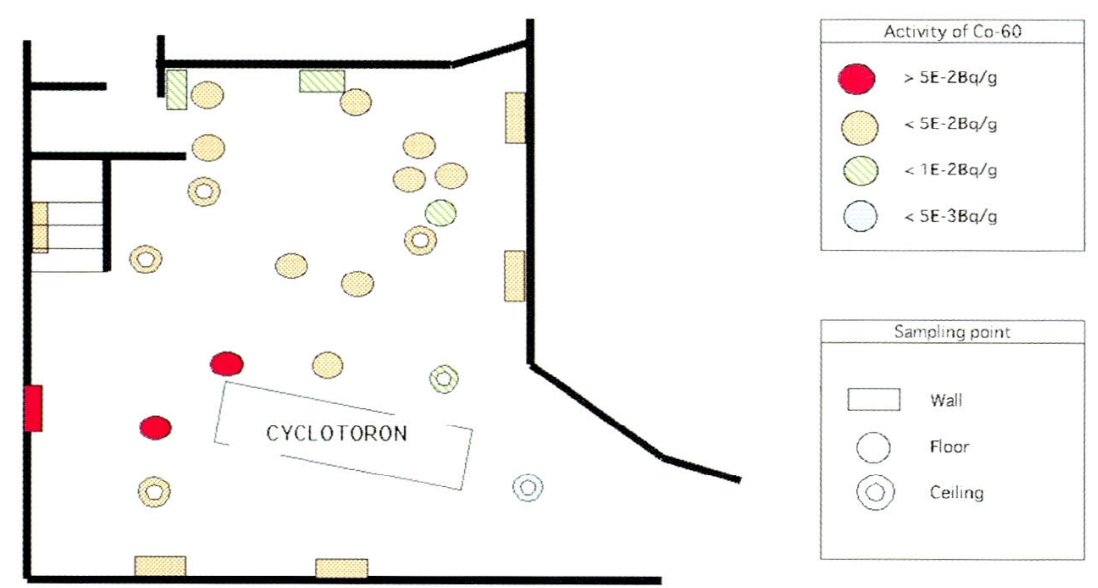

Fig. 5. Specific activity of ${ }^{60} \mathrm{Co}$ in the concrete in the SF-cyclotron building.

trol is to reduce the residual activity of the accelerator components and buildings. The image data contributes to our understanding of neutron propagation and to the effective shiclding of neutrons. Such data will be help to reduce the cost of decommissioning and decontaminating an accelerator facility.

\section{Acknowledgements}

The authors are grateful to Dr. M. Kato and Dr. K. Shikano of NTT photonics laboratory for offering the BAS-1000.

\section{References}

1) Patterson, H. W. and Thomas, R. H.: Accelerator Health Physics, (Academic Press, New York, 1973), p.501.

2) Swanson, W. P.: Radiological Safety Aspects of the Operation of Electron Linear Accelerators, Technical Reports Se- ries No.188 (IAEA, Vienna, 1979).

3) Thomas, R. H. and Stevenson, G. R.: Radiological safety aspects of the operation of proton accelerators. Technical Reports Series No.283 (IAEA, Vienna, 1988)

4) Abe, K.: Appl. Phys., 67, 677(1998).

5) Mori, C. and Matsumura, A.: Nucl. Instr. Methods, A353, 371 (1994).

6) Sonoda, M., Takano, M., Miyahara, J., Kato, H.: Radiology, 148, 833 (1983).

7) Amemiya, Y. and Miyahara, J.: Nature, 336, 89 (1988).

8) Mori, C., Matsumura, A., Suzuki, T., Miyahara, H., Aoyama, T., Nishizawa, K. : Nucl. Instr. Methods, A339, 278 (1994).

9) Masumoto, K., Ohtsuki, T., Miyamoto, Y., Zaidi, J. H., Kajikawa, A., Haba, H. and Sakamoto, K.: J. Radioanal. Nucl. Chem., 239, 495 (1999). 